\title{
Impact of Nanocoating on Micro and Macro Foulers in FRP, Steel and Wooden Materials
}

\author{
S. Archana ${ }^{1 *}$, B. Sundaramoorthy ${ }^{1}$, N. Neethiselvan ${ }^{1}$ and R. Jeyashakila ${ }^{2}$ \\ ${ }^{1}$ Department of Fisheries Technology and Fisheries Engineering, Fisheries College and \\ Research Institute, Tamilnadu Fisheries University, Thoothukudi-628008, India \\ ${ }^{2}$ Department of Fish Quality Assurances and Management, Fisheries College and Research \\ Institute, Tamilnadu Fisheries University, Thoothukudi-628008, India \\ *Corresponding author
}

\begin{abstract}
A B S T R A C T
The field experimental study with test panels was carried out in the jetty of CECRI, Regional centre, located inside the V.O.C. Port at Thoothukudi. Test panels were made from selected boat building

\section{Keywords}

Antifouling,

Nanocoating, Boat building materials, Macrofouling and microfouling

Article Info

Accepted:

26 July 2019

Available Online:

10 August 2019 materials viz., wood, steel and FRP with a size of $(15 \mathrm{~cm} \mathrm{~h} \times 8 \mathrm{~cm} \mathrm{~b}$ and $12 \mathrm{~mm}$ thickness were tied in an iron frame with the dimension of $106.5 \mathrm{~cm} \times 106.5 \mathrm{~cm}$ were allowed to hang at a depth of 1.5 $\mathrm{m}$. Periodical samplings were carried out to study the pattern of microfoulers (diatoms) followed by macrofouling communities. Under microfouling communities, 11 species of diatom identified on the test panels, Nitzchia spp. was found to be most hardy species recorded irrespective of the types of panel and treatments. Steel material showed very poor resistance to serpulid worms despite different treatments. Another important macrofouling community namely barnacles showed progressive increment in terms of numbers during soaking period. Two species of barnacles namely Balanus amphitrite and B. variegates were identified on the test panels, and of these B. amphitrite was found to be the dominant barnacles species. FRP panels showed very poor resistance to foulingby barnacles. Both antifouling treatments viz. (i) nanocoating with copper and (ii) antifouling paint application showed significant effect in controlling mussel and oyster settlement. Two species of mussels namely Perna indica and Anomia spp and two species of oysters viz. Crossesstrea madressensis and Pinctada fucata were recorded on control panels during the study period. However, their settlement was arrested when the plates were given treatment. The commercial grade antifouling paint showed better effect in controlling fouling by ascidians than by copper nanocoating treatment.
\end{abstract}

\section{Introduction}

Biofouling is the deposition and growth of micro and macro organisms on submerged surfaces. Fouling of ship hulls, navigational buoys, underwater equipment, seawater piping systems, industrial or municipal intakes, beach well structures, oil rigs and allied structures has often been reported. In the past few decades, the list of affected structures has expanded. Ships show a 10\% higher fuel consumption caused by increased drag and frictional resistance resulting from hull and propeller fouling. Many marine organisms themselves face the constant problem of being colonized and overgrown by fouling organisms. Immobile plants and animals are generally exposed to biofouling and 
consequent loss of species and community assemblages. Biofouling also promotes corrosion of materials. It is estimated that the marine industry incurs an expenditure of 10 billion sterling pounds a year to combat the situations arising from biofouling worldwide (9).

Biofouling control is a worldwide problem in marine system. One of the earliest methods of solving the problem is to scrape the hulls of ships. When cleaning or scraping becomes time consuming or ineffective, industries turn to perhaps the most of controlling and preventing biofouling namely antifouling coatings. The best method to control the formation of biofouling on submerged surfaces has been found to be the use of anticoating. From the dawn of maritime history, the growth of marine organisms on man-made surfaces, the first attempt to control biofouling goes back to the Greek and Roman civilizations, $700 \mathrm{BC}$, when copper or lead sheathing was used to protect wooden boats (2). Around 1860, ships were built of steel; however copper sheathing could not be used because electrolyte action accelerated the corrosion of the hull ${ }^{(2)}$. This gave the need for alternative methods to protect ships and the dawn of modern paints systems.

A variety of paints was developed mid 1800 s based on the idea of dispersing a toxicant in a polymeric vehicle. Copper-oxide, arsenic, and mercury oxide were popular antifoulants. Recently nanotechnology has been evolved as a tool for the formation of antifouling coating. It has promising future in maritime industries including shipping in controlling the biofouling. Nanocoating has been found to control properties that are responsible for 'non-stick' nature of the surface, such as surface energy, charge, conductivity, porosity, roughness, wettability, friction, physical and chemical reactivity. A European Union research project entitled "Advanced nano structured Surfaces for the Control of Biofouling" has investigated how to prevent the build-up of organisms on surfaces under marine conditions to avoid biofouling. Nanocoating of the metals with antifouling properties have shown positive results for the effective control of fouling in shipping industry in different parts of the world. With this background, an attempt was made to study the effect of copper nano-coating on different boat building materials

\section{Materials and Methods}

\section{Study area}

The study was carried out in the jetty of Central Electro Chemical Research Institute (CECRI), Regional Research Centre, located within the Tuticorin New Port area was chosen for testing the experimental panels. This site has no chance for the mix of freshwater to dilute the seawater so that salinity is reasonably stable except during the monsoon season.

\section{Test panels}

The test panels were made up of Aini wood (Artocarpus hirsutus), Fiber Reinforced Plastic (FRP) and mild steel of width size 15 $\mathrm{cm}$ height, $8 \mathrm{~cm}$ breadth and $12 \mathrm{~mm}$ thickness. The weight of each panel of wood, FRP and steel were $200 \mathrm{gm}, 240 \mathrm{gm}$, and $1 \mathrm{~kg}$, respectively. These test panels were mounted with the help of $4 \mathrm{~mm}$ polypropylene rope in an iron frame having a dimension of $106.5 \mathrm{~cm}$ length, and $106.5 \mathrm{~cm}$ width. Ten panels were mounted in each frame; i.e. five control panels and five coated panels. With the help of a loop provided on the top of the frame, each frame was tied with a $12 \mathrm{~mm}$ polypropylene rope and suspended in the CECRI jetty, inside the Thoothukudi harbour area. All the frames were suspended from the platform at a depth of $1.5 \mathrm{~m}$. 


\section{Sampling schedule}

After immersion in seawater, the study panels were sampled periodically formicro and macrofoulers attachment studies.

For the study of macrofoulers, samplings were carried out after $1^{\text {st }}, 2^{\text {nd }}, 3^{\text {rd }}$ and $4^{\text {th }}$ month from the date of immersion.

\section{Spray coating}

The nano coating was done for wood, steel and FRP panels by spray method.

The synthesized copper nano powder was mixed at the rate of $0.4 \mu \mathrm{g} / \mathrm{L}$ with enamel paint using magnetic stirrer at $60^{\circ} \mathrm{C}$ for $6 \mathrm{~h}$.

Then, the paint was spray coated at 2-4 bar / $30 \mathrm{psi}$ pressure and dried for $24 \mathrm{~h}$ under room temperature.

\section{Antifouling coating}

For comparative studies, all three selected boat-building materials were painted with commercially available antifouling paint (Brand name: NOAH Marine Paints, Cochin).

\section{Identification of Micro and Macro foulers}

Micro and macro foulers like protozoa, diatoms, were observed under laser microscopy method and barnacles, bivalves, crabs, etc. were identified using the guideline of Fouling Organisms and Instruction Regarding the Docking Report (1942),

Instructions Regarding the Docking Report and Guide to Fouling Organisms (1944) and Fouling of Ships' Bottoms: Identification of Marine Growths (1944).

The total numbers of macro-foulers were calculated and tabulated on each sampling.

\section{Results and Discussion}

\section{Diatom species recorded on various test panels}

Among the three types of panels tested, the wooden control panel harbor more number of diatom species during the $90^{\text {th }}$ day of inspection. The control wooden panel, control steel panel and control FRP panel had 7, 5 and 2 settlers respectively. Regarding antifouling coated panels; there was a domination by single species irrespective of the type of panel. However, Vorticella spp dominated on antifouling coated wooden panel while Nitzchia spp dominated over antifouling coated steel panel and antifouling coated FRP panel. In the case of nanocoated panels, on $90^{\text {th }}$ day of observation, clear-cut impact was noticeable on nanocoated FRP panel, as it was dominated only by single species. However, it was not significant on nanocoated wooden and nanocoated steel panels as 3 and 4 species were recorded. As the soaking duration increases from $90^{\text {th }}$ to $150^{\text {th }}$ day, a clear-cut reduction in species diversity was noticed with respect to wooden control panel and steel control panel. However, a reverse phenomenon was observed with respect to FRP control panel. In the case of control wooden panel, the number of species reduced from 7 to 2 from $90^{\text {th }}$ day of observation to $150^{\text {th }}$ day of observation. It was from 5 to 1 in the case of control steel panel. Regarding control FRP panel, an increase was noticed from 2 genera to 5 genera (Table 1). Regarding species succession due to antifouling painting test panels on various effect was found to be highly pronounced on steel panel as it allowed single genus namely, Nitzchia spp alone from $90^{\text {th }}$ day to $150^{\text {th }}$ day. However, when antifouling paint was coated over wooden and FRP panels, it could retain single genus distribution over the panel surface up to $120^{\text {th }}$ day and thereafter the number of species increased to 4 with same 
composition both in wood and FRP panel on $150^{\text {th }}$ day of observation. Nano-coating was found to have noticeable impact with respect to FRP panel up to 90 days thereafter the species diversity increased with the representation of 4 genera on $150^{\text {th }}$ day of observation.

Regarding nano-coating over wooden and steel panel no visible impact could be seen as evidenced through the contribution of same number of genera throughout the study period during different intervals of sampling. The study clearly indicated that the genus Nitzchia as the most hardy genus of diatom both in antifouling paint and nanocoated with copper. (Table 2).

Serpulid worm recorded on various test panels

Among the three types of panel tested, wooden panels both treated and control did not show any occurrence of serpulid worm throughout the study period. In the case of FRP panel, occurrence of serpulid worms was arrested throughout the study period by antifouling painting and nano coating of copper.

However, control FRP panel showed the highest occurrence of five numbers per panel throughout the study period. It was evident that soaking duration did not have any impact and abundance of serpulid worm on different panels, which showed its occurrence (control steel panel, anti-fouling painted steel panel, nano coated steel panel).

Among the materials tested, steel was prone to infestation by serpulid worm was noticed both on control and treated panels. Both antifouling painting and nano coating of copper gave negative impacts as evidenced through higher abundance of this worm over antifouling painted and nano coated panels compared to control panels (Table 3).

\section{Barnacle species recorded on various test panels}

The details on various barnacle species recorded on various test panels during different study period are given in (Table 4). From the result of the analysis of barnacle settlement over different test panels, following inferences could be arrived.

There was progressive increment in the number of barnacle with the duration of soaking. Balanus amphitrite was found to be the hardiest species as its distribution could be noted in all the panels irrespective of the type and antifouling treatment

Balanus variegates also showed occurrence in all the types of panels tested. However, after $120^{\text {th }}$ day of observation in the case of nano coated FRP, nano coated wood, nano coated steel panels. Further, this species showed about 25 percent contribution in terms of number whenever it co-occurred with the Balanus amphitrite.

Among the control panels, the barnacle settlement was higher over FRP panel. With respect to steel control panel there was a sudden increment after the second sampling. Regarding control panels, the barnacle settlement was lower in wooden panels in relation to FRP and steel panels.

Irrespective the types of panels anti-fouling painting could arrest the settlement of Balanus variegates upto $90^{\text {th }}$ day of soaking. Though anti-fouling painting could have notable impact on the settlement of fouler over different types of material studied, the effect was lower in FRP followed by steel and wood.

Nano coating could prolonged the nonsettlement period of Balanus variegates on the three types of panel tested upto $120^{\text {th }}$ day while it was $90^{\text {th }}$ day by anti-fouling painting. 
Nano coating was found to be more effective on steel compared to wood and FRP (Table 4).

Mussel species recorded on various test panels

The fouling of Perna indica and Anomia spp observed only on control panels irrespective of its type. Infestation both by Perna indica and Anomia spp could be observed over control wooden and control FRP panel. Further, the mussel infestation could be observed in over control wooden and FRP panel during the second sampling. There was a progressive increase of the two mussel species over control FRP panel, while the number remains same in the case of control wooden panel. There was a infestation by Perna indica over control steel panel starting from the first sampling however with no change in population density over the panel (Table 5).

Oyster species recorded on various test panels

Fouling by the edible oyster Crossastrea madrasensis and Pinctada fucata could be observed only on control steel panel and control FRP Panel. In these two types of panels progressive increase with the duration of soaking could be observed on control FRP Panel. In the case of control steel panel increment in number of edible oyster and pearl oyster could be observed only after $120^{\text {th }}$ day (Table 6).

\section{Ascidians species recorded on various test panels}

Among the three types of test panels studied, the attachment of ascidians was found to be higher over FRP control panels. Its number got reduced when treated with nano coating with copper. However, anti-fouling painting did not show any significant impact on FRP panel. Similar impact was observed with respect to wooden test panel. Steel panel were found to be resistant to infestation by ascidians. Ascidians did not show any significant increase with duration of soaking with respect to different types of panel studied (Table 7).

\section{Diatom species recorded on various test panels}

As far as diatom settlement is concerned, there were clear-cut differences noticed between the control and treated test panels irrespective of materials. Among the controls, wooden panels were more attracted by diatoms. Among the treated, antifouling paint coated panels showed less number of diatom settlements than that of copper nanocoated. Among the species settled, Nitzchia spp was found to be dominant and found in majority of panels studied. (3) studied the fouling diatom from offshore waters of Bombay and he recorded 58 species of fouling diatoms on aluminium panels. Also he recorded Navicula, Coscinodiscus, Rhizosolenia and Chetoceros dominated in the biodiversity of fouling diatoms. In the west coast of India, another study was carried out by ${ }^{(6)}$ using mild steel, aluminium FRP and glass panels and he recorded 92 species from Waghotana estuary. (5) investigated the diatoms of Goa Coast and found that Navicula, Synedra and Nitzchia species were dominated the samples. As most of these works were carried out in the west Coast of India, the species composition and population are showing great deviation from the present work. The present study reveals that the genus Nitzchia is most hardly genus of diatom for both antifouling painted and copper nanocoated panels. The nanocoated wooden, steel panels showed no visible impact on controlling the diatoms, but the nanocoated FRP showed some impact in the early phase of study and subsequently with more number of genera of diatoms. 
Table.1 Diatom species recorded on various test panels

\begin{tabular}{|c|c|c|c|c|}
\hline $\begin{array}{l}\text { S. } \\
\text { No }\end{array}$ & Panel type & $\begin{array}{l}\text { First sampling } \\
\left(90^{\text {th }} \text { day }\right)\end{array}$ & $\begin{array}{l}\text { Second sampling } \\
\left(\mathbf{1 2 0}^{\text {th }} \text { day }\right)\end{array}$ & $\begin{array}{l}\text { Third sampling } \\
\left(150^{\text {th }} \text { day }\right)\end{array}$ \\
\hline \multirow[t]{3}{*}{ 1) } & a. Wood control & $\begin{array}{ll}\text { 1. } & \text { Chlorella spp. } \\
\text { 2. } & \text { Coscinodiscus spp. } \\
\text { 3. } & \text { Fragilaria spp } \\
\text { 4. } & \text { Navicula spp. } \\
\text { 5. } & \text { Nitzchia spp. } \\
\text { 6. } & \text { Stentor spp } \\
\text { 7. } & \text { Vorticella spp. }\end{array}$ & $\begin{array}{l}\text { Bacilaria spp. } \\
\text { Chlorella spp. } \\
\text { Synedra spp. } \\
\text { Vorticella spp. }\end{array}$ & $\begin{array}{l}\text { Nitzchia spp. } \\
\text { Synedra spp. }\end{array}$ \\
\hline & $\begin{array}{l}\text { b. Woodantifoulin } \\
\text { g paint coated }\end{array}$ & Vorticella spp. & Nitzchia spp. & $\begin{array}{ll}\text { 1. } & \text { Chlorella spp. } \\
\text { 2. } & \text { Navicula spp } \\
\text { 3. } & \text { Nitzchia spp. } \\
\text { 4. } & \text { Synedra spp. }\end{array}$ \\
\hline & $\begin{array}{l}\text { c.Wood } \\
\text { nanocoated }\end{array}$ & $\begin{array}{ll}\text { 1. } & \text { Chlorella spp. } \\
\text { 2. } & \text { Netrium spp. } \\
\text { 3. } & \text { Nitzchia spp. }\end{array}$ & $\begin{array}{l}\text { Navicula spp. } \\
\text { Nitzchia spp. } \\
\text { Synedra spp. }\end{array}$ & $\begin{array}{ll}\text { 1. } & \text { Bacillaria spp. } \\
\text { 2. } & \text { Navicula spp. } \\
\text { 3. } & \text { Nitzchia spp. } \\
\text { 4. } & \text { Synedra spp. }\end{array}$ \\
\hline \multirow[t]{3}{*}{ 2) } & a.Steel control & $\begin{array}{ll}\text { 1. } & \text { Bacillaria spp. } \\
\text { 2. } & \text { Chlorella spp. } \\
\text { 3. } & \text { Fragilaria spp. } \\
\text { 4. } & \text { Netrium spp. } \\
\text { 5. } & \text { Sphaeroma spp. }\end{array}$ & $\begin{array}{ll}\text { 1. } & \text { Fragilaria spp. } \\
\text { 2. } & \text { Navicula spp. } \\
\text { 3. } & \text { Sphaeroma } \\
\text { spp. } & \end{array}$ & Synedra spp. \\
\hline & $\begin{array}{l}\text { b.Steel } \\
\text { antifouling paint } \\
\text { coated }\end{array}$ & Nitzchia spp. & Nitzchia spp. & Nitzchia spp. \\
\hline & $\begin{array}{l}\text { c.Steel } \\
\text { nanocoated }\end{array}$ & $\begin{array}{ll}\text { 1. } & \text { Chlorella spp. } \\
\text { 2. } & \text { Fragilaria spp. } \\
\text { 3. } & \text { Netrium spp. } \\
\text { 4. } & \text { Nitzchia spp. }\end{array}$ & $\begin{array}{ll}\text { 1. } & \text { Nitzchia spp. } \\
\text { 2. } & \text { Synedra spp. }\end{array}$ & $\begin{array}{ll}\text { 1. } & \text { Navicula spp. } \\
\text { 2. } & \text { Nitzchia spp. } \\
\text { 3. } & \text { Synedra spp. }\end{array}$ \\
\hline
\end{tabular}




\begin{tabular}{|c|c|c|c|c|c|c|c|}
\hline 3) & a.FRP control & $\begin{array}{l}1 . \\
2 .\end{array}$ & $\begin{array}{l}\text { Navicula spp. } \\
\text { Nitzchia spp. }\end{array}$ & $\begin{array}{l}1 . \\
2 . \\
3 . \\
4 . \\
5 .\end{array}$ & $\begin{array}{l}\text { Bacillaria spp. } \\
\text { Chlorella spp. } \\
\text { Netrium spp. } \\
\text { Nitzchia spp. } \\
\text { Synedra spp. }\end{array}$ & $\begin{array}{l}1 . \\
2 . \\
3 . \\
4 . \\
5 .\end{array}$ & $\begin{array}{l}\text { Bacillaria spp. } \\
\text { Navicula spp. } \\
\text { Netrium spp. } \\
\text { Nitzchia spp. } \\
\text { Synedra spp. }\end{array}$ \\
\hline & $\begin{array}{l}\text { b.FRP antifouling } \\
\text { paint coated }\end{array}$ & 1. & Nitzchia spp. & 1. & Nitzchia spp. & $\begin{array}{l}1 . \\
2 . \\
3 . \\
4 .\end{array}$ & $\begin{array}{l}\text { Chlorella spp } \\
\text { Navicula spp. } \\
\text { Nitzchia spp. } \\
\text { Synedra spp. }\end{array}$ \\
\hline & $\begin{array}{l}\text { c.FRP } \\
\text { nanocoated }\end{array}$ & 1. & Nitzchia spp. & $\begin{array}{l}1 . \\
2 . \\
3 .\end{array}$ & $\begin{array}{l}\text { Navicula spp. } \\
\text { Nitzchia spp. } \\
\text { Synedra spp. }\end{array}$ & $\begin{array}{l}1 . \\
2 . \\
3 . \\
4 .\end{array}$ & $\begin{array}{l}\text { Bacillaria spp. } \\
\text { Navicula spp. } \\
\text { Nitzchia spp. } \\
\text { Synedra spp. }\end{array}$ \\
\hline
\end{tabular}

Table.2 Analysis of variance of diatom species recorded on various test panels

\begin{tabular}{|l|c|c|c|c|c|}
\hline $\begin{array}{l}\text { Source of } \\
\text { Variation }\end{array}$ & $\begin{array}{l}\text { Sum of } \\
\text { Square }\end{array}$ & $\begin{array}{l}\text { Degree of } \\
\text { Freedom }\end{array}$ & $\begin{array}{l}\text { Mean } \\
\text { Sum of } \\
\text { Square }\end{array}$ & F- value & $\begin{array}{l}\text { P-value } \\
\text { (P<0.05) }\end{array}$ \\
\hline $\begin{array}{l}\text { Between } \\
\text { Controls }\end{array}$ & 73.33333 & 24 & 3.055556 & 0.339394 & 0.715565 \\
\hline $\begin{array}{l}\text { Between } \\
\text { treatments }\end{array}$ & 2.074074 & 2 & 1.037037 & -- & - \\
\hline Error & 9.804239 & 8 & 1.22553 & - & - \\
\hline Total & & & & & \\
\hline & 55.2028 & 17 & - & - & - \\
\hline
\end{tabular}


Table.3 Serpulid worm and their abundance on various test panel

\begin{tabular}{|c|c|c|c|c|c|c|c|}
\hline \multirow{2}{*}{$\begin{array}{l}\text { S. } \\
\text { No }\end{array}$} & \multirow[t]{2}{*}{ Panel type } & \multicolumn{2}{|c|}{ First sampling } & \multicolumn{2}{|c|}{ Second sampling } & \multicolumn{2}{|c|}{ Third sampling } \\
\hline & & $\begin{array}{c}\text { Species wise } \\
\text { number per } \\
\text { panel }\end{array}$ & $\begin{array}{c}\text { Total } \\
\text { number } \\
\text { per panel }\end{array}$ & $\begin{array}{c}\text { Species wise } \\
\text { number per } \\
\text { panel }\end{array}$ & $\begin{array}{c}\text { Total } \\
\text { number } \\
\text { per } \\
\text { panel }\end{array}$ & $\begin{array}{c}\text { Species wise } \\
\text { number per } \\
\text { panel }\end{array}$ & $\begin{array}{c}\text { Total } \\
\text { number } \\
\text { per panel }\end{array}$ \\
\hline \multirow[t]{3}{*}{ 1) } & a. Wood control & NIL & NIL & NIL & NIL & NIL & NIL \\
\hline & $\begin{array}{l}\text { b. Wood } \\
\text { antifouling paint } \\
\text { coated }\end{array}$ & NIL & NIL & NIL & NIL & NIL & NIL \\
\hline & $\begin{array}{l}\text { c.Wood nano- } \\
\text { coated }\end{array}$ & NIL & NIL & NIL & NIL & NIL & NIL \\
\hline \multirow[t]{3}{*}{ 2) } & a.Steel control & $\begin{array}{c}\text { Serpulid } \\
\text { worm }\end{array}$ & 2 & Serpulid worm & 2 & Serpulid worm & 2 \\
\hline & $\begin{array}{l}\text { b. Steel } \\
\text { antifouling paint } \\
\text { coated }\end{array}$ & $\begin{array}{l}\text { Serpulid } \\
\text { worm }\end{array}$ & 4 & Serpulid worm & 4 & Serpulid worm & 4 \\
\hline & $\begin{array}{l}\text { c.Steel nano- } \\
\text { coated }\end{array}$ & $\begin{array}{l}\text { Serpulid } \\
\text { worm }\end{array}$ & 3 & Serpulid worm & 3 & Serpulid worm & 3 \\
\hline \multirow[t]{3}{*}{ 3) } & a.FRP control & $\begin{array}{c}\text { Serpulid } \\
\text { worm }\end{array}$ & 5 & Serpulid worm & 5 & Serpulid worm & 5 \\
\hline & $\begin{array}{l}\text { b. FRP } \\
\text { antifouling paint } \\
\text { coated }\end{array}$ & NIL & NIL & NIL & NIL & NIL & NIL \\
\hline & $\begin{array}{l}\text { c. FRP nano- } \\
\text { coated }\end{array}$ & NIL & NIL & NIL & NIL & NIL & NIL \\
\hline
\end{tabular}


Table.4 Barnacle species and their abundance on various test panels

\begin{tabular}{|c|c|c|c|c|c|c|c|c|c|c|}
\hline \multirow{2}{*}{ S. No } & \multirow{2}{*}{ Panel type } & \multicolumn{3}{|c|}{ First sampling } & \multicolumn{3}{|c|}{ Second sampling } & \multicolumn{3}{|c|}{ Third sampling } \\
\hline & & $\begin{array}{l}\text { Species wise number } \\
\text { per panel }\end{array}$ & $\%$ & $\begin{array}{l}\text { Total } \\
\text { number per } \\
\text { panel }\end{array}$ & $\begin{array}{l}\text { Species wise } \\
\text { number per panel }\end{array}$ & $\%$ & $\begin{array}{l}\text { Total } \\
\text { number per } \\
\text { panel }\end{array}$ & $\begin{array}{l}\text { Species wise } \\
\text { number per panel }\end{array}$ & $\%$ & $\begin{array}{l}\text { Total number } \\
\text { per panel }\end{array}$ \\
\hline \multirow[t]{6}{*}{ 1) } & \multirow[t]{2}{*}{$\begin{array}{l}\text { a. Wood } \\
\text { control }\end{array}$} & Balanus amphitrite-18 & 75 & \multirow[t]{2}{*}{24} & $\begin{array}{l}\text { Balanus amphitrite- } \\
60\end{array}$ & 76 & \multirow[t]{2}{*}{78} & $\begin{array}{l}\text { Balanus amphitrite- } \\
99\end{array}$ & 76 & \multirow[t]{2}{*}{130} \\
\hline & & Balanus variegates- 6 & 25 & & $\begin{array}{c}\text { Balanus variegates- } \\
18\end{array}$ & 24 & & $\begin{array}{l}\text { Balanus variegates- } \\
31\end{array}$ & 24 & \\
\hline & \multirow[t]{2}{*}{$\begin{array}{l}\text { b.Wood antifouling } \\
\text { paint coated }\end{array}$} & Balanus amphitrite-12 & 100 & \multirow[t]{2}{*}{12} & $\begin{array}{c}\text { Balanus amphitrite- } \\
14\end{array}$ & 74 & \multirow[t]{2}{*}{19} & $\begin{array}{c}\text { Balanus amphitrite- } \\
24\end{array}$ & 75 & \multirow[t]{2}{*}{32} \\
\hline & & & & & Balanus variegates -5 & 26 & & Balanus variegates-8 & 25 & \\
\hline & \multirow[t]{2}{*}{ c.Wood nano-coated } & \multirow[t]{2}{*}{ Balanus amphitrite-16 } & \multirow[t]{2}{*}{100} & \multirow[t]{2}{*}{16} & \multirow[t]{2}{*}{$\begin{array}{l}\text { Balanus amphitrite- } \\
108\end{array}$} & \multirow[t]{2}{*}{100} & \multirow[t]{2}{*}{108} & $\begin{array}{c}\text { Balanus amphitrite- } \\
108\end{array}$ & 75 & \multirow[t]{2}{*}{144} \\
\hline & & & & & & & & $\begin{array}{l}\text { Balanus variegates- } \\
36\end{array}$ & 25 & \\
\hline \multirow[t]{6}{*}{ 2) } & \multirow[t]{2}{*}{ a.Steel control } & Balanus amphitrite- 3 & 75 & \multirow[t]{2}{*}{4} & $\begin{array}{l}\text { Balanus amphitrite- } \\
18\end{array}$ & 75 & \multirow[t]{2}{*}{24} & $\begin{array}{c}\text { Balanus amphitrite- } \\
139\end{array}$ & 75 & \multirow[t]{2}{*}{185} \\
\hline & & Balanus variegates-1 & 25 & & $\begin{array}{l}\text { Balanus variegatecs- } \\
6\end{array}$ & 25 & & $\begin{array}{l}\text { Balanus variegates- } \\
46\end{array}$ & 25 & \\
\hline & \multirow[t]{2}{*}{$\begin{array}{l}\text { b.Steel antifouling } \\
\text { paint coated }\end{array}$} & Balanus Amphitrite - 6 & 100 & \multirow[t]{2}{*}{6} & $\begin{array}{l}\text { Balanus amphitrite- } \\
23\end{array}$ & 76 & \multirow[t]{2}{*}{29} & $\begin{array}{l}\text { Balanus amphitrite- } \\
45\end{array}$ & 74 & \multirow[t]{2}{*}{61} \\
\hline & & & & & Balanus variegates-6 & 24 & & $\begin{array}{c}\text { Balanus variegates- } \\
16\end{array}$ & 26 & \\
\hline & \multirow[t]{2}{*}{ c.Steel nano-coated } & Balanus amphitrite-12 & 100 & \multirow[t]{2}{*}{12} & $\begin{array}{l}\text { Balanus amphitrite- } \\
\qquad 12\end{array}$ & 100 & \multirow[t]{2}{*}{12} & $\begin{array}{l}\text { Balanus amphitrite- } \\
55\end{array}$ & 74 & 74 \\
\hline & & & & & & & & $\begin{array}{c}\text { Balanus variegates- } \\
19\end{array}$ & 26 & \\
\hline 3) & a.FRP control & Balanus amphitrite-34 & 75 & 46 & $\begin{array}{l}\text { Balanus amphitrite- } \\
64\end{array}$ & 75 & 85 & $\begin{array}{l}\text { Balanus amphitrite- } \\
\qquad 120\end{array}$ & 75 & 160 \\
\hline & & Balanus variegates -12 & 25 & & $\begin{array}{c}\text { Balanus variegates- } \\
21\end{array}$ & 25 & & $\begin{array}{c}\text { Balanus variegates- } \\
40\end{array}$ & 25 & \\
\hline & $\begin{array}{l}\text { b.FRP antifouling } \\
\text { paint coated }\end{array}$ & Balanus amphitrite-29 & 100 & 29 & $\begin{array}{l}\text { Balanus amphitrite- } \\
29\end{array}$ & 76 & 38 & $\begin{array}{l}\text { Balanus amphitrite- } \\
101\end{array}$ & 75 & 135 \\
\hline & & & & & Balanus variegates-9 & 24 & & $\begin{array}{c}\text { Balanus variegates- } \\
34\end{array}$ & 25 & \\
\hline & $\begin{array}{l}\text { c.FRP } \\
\text { nanocoatedpanel }\end{array}$ & Balanus amphitrite-15 & 100 & 15 & $\begin{array}{l}\text { Balanus amphitrite- } \\
111\end{array}$ & 100 & 111 & $\begin{array}{l}\text { Balanus amphitrite- } \\
\qquad 121\end{array}$ & 75 & 162 \\
\hline & & & & & & & & $\begin{array}{l}\text { Balanus variegates- } \\
41\end{array}$ & 25 & \\
\hline
\end{tabular}


Table.5 Mussel species and their abundance on various test panels

\begin{tabular}{|c|c|c|c|c|c|c|c|}
\hline \multirow[b]{2}{*}{$\begin{array}{l}\text { S. } \\
\text { No }\end{array}$} & \multirow[b]{2}{*}{ Panel type } & \multicolumn{2}{|c|}{ First sampling } & \multicolumn{2}{|c|}{ Second sampling } & \multicolumn{2}{|c|}{ Third sampling } \\
\hline & & $\begin{array}{l}\text { Species wise } \\
\text { number per } \\
\text { panel }\end{array}$ & $\begin{array}{c}\text { Total } \\
\text { number per } \\
\text { panel }\end{array}$ & $\begin{array}{c}\text { Species wise } \\
\text { number per } \\
\text { panel }\end{array}$ & $\begin{array}{c}\text { Total number } \\
\text { per panel }\end{array}$ & $\begin{array}{c}\text { Species wise } \\
\text { number per } \\
\text { panel }\end{array}$ & $\begin{array}{c}\text { Total } \\
\text { number } \\
\text { per panel }\end{array}$ \\
\hline 1) & a.Wood control & NIL & NIL & $\begin{array}{l}\text { Perna indica-1 } \\
\text { Anomia spp-1 }\end{array}$ & 2 & $\begin{array}{l}\text { Perna indica-1 } \\
\text { Anomia spp-1 }\end{array}$ & 2 \\
\hline & $\begin{array}{l}\text { b. Wood antifouling paint } \\
\text { coated }\end{array}$ & NIL & NIL & NIL & NIL & NIL & NIL \\
\hline & c. Wood nano-coated & NIL & NIL & NIL & NIL & NIL & NIL \\
\hline 2) & a. Steel control & Perna indica-2 & 2 & Perna indica-2 & 2 & Perna indica-2 & 2 \\
\hline & $\begin{array}{l}\text { b. Steel antifouling paint } \\
\text { coated }\end{array}$ & NIL & NIL & NIL & NIL & NIL & NIL \\
\hline & c. Steel nano-coated & NIL & NIL & NIL & NIL & NIL & NIL \\
\hline 3) & a. FRP control & NIL & NIL & $\begin{array}{l}\text { Perna indica-2 } \\
\text { Anomia spp-2 }\end{array}$ & 4 & $\begin{array}{l}\text { Perna indica-6 } \\
\text { Anomia spp-4 }\end{array}$ & 10 \\
\hline & $\begin{array}{l}\text { b.FRP antifouling paint } \\
\text { coated }\end{array}$ & NIL & NIL & NIL & NIL & NIL & NIL \\
\hline & c. FRP nanocoated panel & NIL & NIL & NIL & NIL & NIL & NIL \\
\hline
\end{tabular}


Table.6 Oyster species and their abundance on various test panels

\begin{tabular}{|c|c|c|c|c|c|c|c|}
\hline \multirow{2}{*}{$\begin{array}{l}\text { S. } \\
\text { No }\end{array}$} & \multirow[t]{2}{*}{ Panel type } & \multicolumn{2}{|c|}{ First sampling } & \multicolumn{2}{|c|}{ Second sampling } & \multicolumn{2}{|c|}{ Third sampling } \\
\hline & & $\begin{array}{c}\text { Species wise } \\
\text { number per panel }\end{array}$ & $\begin{array}{c}\text { Total } \\
\text { number per } \\
\text { panel }\end{array}$ & $\begin{array}{c}\text { Species wise } \\
\text { number per panel }\end{array}$ & $\begin{array}{c}\text { Total } \\
\text { number } \\
\text { per panel }\end{array}$ & $\begin{array}{l}\text { Species wise } \\
\text { number per } \\
\text { panel }\end{array}$ & $\begin{array}{l}\text { Total number } \\
\text { per panel }\end{array}$ \\
\hline \multirow[t]{3}{*}{ 1) } & a. Wood control & NIL & NIL & NIL & NIL & NIL & NIL \\
\hline & $\begin{array}{l}\text { b. Wood antifouling } \\
\text { paint coated }\end{array}$ & NIL & NIL & NIL & NIL & NIL & NIL \\
\hline & c. Wood nano-coated & NIL & NIL & NIL & NIL & NIL & NIL \\
\hline \multirow[t]{3}{*}{ 2) } & a. Steel control & $\begin{array}{c}\text { Crossastrea } \\
\text { madrasensis-4 } \\
\text { Pinctada fucata-6 }\end{array}$ & 10 & $\begin{array}{c}\text { Crossastrea } \\
\text { madrasensis-4 } \\
\text { Pinctada fucata-6 }\end{array}$ & 10 & $\begin{array}{c}\text { Crossastrea } \\
\text { madrasensis-6 } \\
\text { Pinctada } \\
\text { fucata-8 }\end{array}$ & 14 \\
\hline & $\begin{array}{l}\text { b.Steel antifouling } \\
\text { paint coated }\end{array}$ & NIL & NIL & NIL & NIL & NIL & NIL \\
\hline & c. Steel nano-coated & NIL & NIL & NIL & NIL & NIL & NIL \\
\hline \multirow[t]{3}{*}{ 3) } & a. FRP control & $\begin{array}{c}\text { Crossastrea } \\
\text { madrasensis-1 } \\
\text { Pinctada fucata-1 }\end{array}$ & 2 & $\begin{array}{c}\text { Crossastrea } \\
\text { madrasensis-2 } \\
\text { Pinctada fucata-4 }\end{array}$ & 6 & $\begin{array}{c}\text { Crossastrea } \\
\text { madrasensis-6 } \\
\text { Pinctada } \\
\text { fucata-4 }\end{array}$ & 10 \\
\hline & $\begin{array}{l}\text { b. FRP antifouling } \\
\text { paint coated }\end{array}$ & NIL & NIL & NIL & NIL & NIL & NIL \\
\hline & c. FRP nanocoated & NIL & NIL & NIL & NIL & NIL & NIL \\
\hline
\end{tabular}


Table.7 Ascidians species and their abundance on various test panels

\begin{tabular}{|c|c|c|c|c|c|c|c|}
\hline \multirow{2}{*}{$\begin{array}{l}\text { S. } \\
\text { No }\end{array}$} & \multirow[t]{2}{*}{ Panel type } & \multicolumn{2}{|c|}{ First sampling } & \multicolumn{2}{|c|}{ Second sampling } & \multicolumn{2}{|c|}{ Third sampling } \\
\hline & & $\begin{array}{c}\text { Species wise } \\
\text { Number per } \\
\text { panel }\end{array}$ & $\begin{array}{c}\text { Total } \\
\text { number } \\
\text { per panel }\end{array}$ & $\begin{array}{l}\text { Species wise } \\
\text { number per } \\
\text { panel }\end{array}$ & $\begin{array}{c}\text { Total } \\
\text { number } \\
\text { per } \\
\text { panel }\end{array}$ & $\begin{array}{c}\text { Species wise } \\
\text { number per panel }\end{array}$ & $\begin{array}{c}\text { Total } \\
\text { number } \\
\text { per } \\
\text { panel }\end{array}$ \\
\hline \multirow[t]{3}{*}{ 1) } & a. Wood control & $\begin{array}{l}\text { Diplosoma } \\
\text { spp-2 }\end{array}$ & 2 & Diplosoma spp-1 & 2 & $\begin{array}{c}\text { Diplosoma spp-1 } \\
\text { Phallusia spp-1 }\end{array}$ & 2 \\
\hline & $\begin{array}{l}\text { b. Wood } \\
\text { antifouling } \\
\text { paint coated }\end{array}$ & NIL & NIL & NIL & NIL & NIL & NIL \\
\hline & $\begin{array}{l}\text { c. Wood nano- } \\
\text { coated }\end{array}$ & $\begin{array}{l}\text { Didemnum } \\
\text { spp-1 } \\
\text { Diplosoma } \\
\text { spp-1 }\end{array}$ & 2 & $\begin{array}{l}\text { Didemnum spp-1 } \\
\text { Diplosoma spp-1 }\end{array}$ & 2 & $\begin{array}{l}\text { Didemnum spp-1 } \\
\text { Diplosoma spp-1 }\end{array}$ & 2 \\
\hline \multirow[t]{3}{*}{ 2) } & a. Steel control & NIL & NIL & NIL & NIL & NIL & NIL \\
\hline & $\begin{array}{l}\text { b. Steel } \\
\text { antifouling } \\
\text { paint coated }\end{array}$ & NIL & NIL & NIL & NIL & NIL & NIL \\
\hline & $\begin{array}{l}\text { c. Steel nano- } \\
\text { coated }\end{array}$ & NIL & NIL & NIL & NIL & NIL & NIL \\
\hline \multirow[t]{3}{*}{ 3) } & a. FRP control & $\begin{array}{l}\text { Didemnum } \\
\text { spp-1 }\end{array}$ & 1 & Didemnum spp-1 & 1 & $\begin{array}{l}\text { Didemnum spp-1 } \\
\text { Diplosoma spp-1 } \\
\text { Phallusia spp-1 }\end{array}$ & 3 \\
\hline & $\begin{array}{l}\text { b.FRP } \\
\text { antifouling } \\
\text { paint coated }\end{array}$ & NIL & NIL & NIL & NIL & NIL & NIL \\
\hline & $\begin{array}{l}\text { c. FRP nano- } \\
\text { coated }\end{array}$ & $\begin{array}{l}\text { Didemnum } \\
\text { spp-1 }\end{array}$ & 1 & Didemnum spp-1 & 1 & Didemnum spp-1 & 1 \\
\hline
\end{tabular}

Serpulid worm recorded on various test panels

The control and treated (antifouling painted and nanocoated) wooden panels were found with serpulid wormfouling throughout the study period. But steel panels were found to be recorded with more number of serpulid worms irrespective of control and treated. With regard to FRP, the treated panels showed good effect on controlling serpulid worm fouling when compared to that of control. (12) studied the impact of wood preservatives on the settlement of growth of fouling organisms in Krishnapattinam. $\mathrm{He}$ found that the serpulid worms were found to be negligible on the treated panels; however, their settlement was heavy on control panels.

\section{Barnacles recorded on various test panels}

The barnacle fouling on both control treated panels of different boats building materials are presented in the (Table 4). In general, the barnacle fouling increased progressively with duration of soaking, irrespective of materials selected for study. Only two species of barnacles viz. B.amphitrite and B. variegates were recorded throughout the study period of these two species B.amphitrite was found to 
be present in all the panels irrespective of the types and antifouling treatments. The other species B.variegates showed one fourth of the fouling barnacles by numbers and remaining portion by B.amphitrite. High degree of barnacle fouling was observed in FRP control panels, when compared to other two boat building materials. However, the untreated wooden panels showed comparatively less barnacle fouling.

It could be inferred that the antifouling paint treated panels had effectiveness upto $90^{\text {th }}$ day of soaking. But, in the case of copper nanocoated panels they showed good antifouling effect upto 120 days of soaking, particularly for B.variegates. ${ }^{(4)}$ found that B.amphitrite is the dominant barnacle species in Thoothukudi coast and recorded year round spawning. The high nutrient load of the seawater promotes massive growth of algae that serve as food for the barnacles along Thoothukudi coast. This is in accordance with the results of the present study. (4) also confirmed that one-month static immersion is more than adequate for barnacle attachment to occur with visible, measureable adult barnacles.

Regarding the species composition of barnacles, the present study showed very less biodiversity with only two species. (7) compared the marine fouling community in the Eastern Harbour of Alexandria, Egypt with four decades of previous studies and he found that there was remarkable variation in number and diversity of fouling communities, due to many factors, of which nutrient enrichment is the most important. A study was conducted in Vellar Estuary mangrove areas by ${ }^{(10)}$ also confirmed the $B$. amphitrite dominated among other fouling species. In Mumbai Harbour four species of barnacles were also reported by ${ }^{(11)}$. Four species of barnacles were reported in Pondicherry Coast of Tamil Nadu by ${ }^{(1)}$. When compared to previous studies, the present study records only two species of barnacles this may be due to poor food availability in the Thoothukudi port area, during study period.

\section{Mussel species recorded on various test panels}

As far as mussel fouling is concerned, there was no much species involved in the fouling of test panels. Both the antifouling treatments tested for this study showed good impact on controlling the mussel fouling. Two species namely Perna indica and Anomia spp were recorded on controls of different boat building materials and the number of individual increased progressively.

In Edaiyur Backwaters of Kalpakkam, Tamil $\mathrm{Nadu}$, both green and brown mussels were recorded as foulers ${ }^{(8)}$. In addition, the authors recorded that between the two mussels, green mussels dominated throughout the year. (11) recorded very poor molluscan settlement on the test panels with no species under mussel group. While studying the distribution of macro fouling community in the coastal region of Pondichery. ${ }^{(1)}$ recorded only two species of mussels namely Perna visidis and Modiolus spp.

\section{Oyster species recorded on various test panels}

Two species of oysters' viz. C.madrasensis and Pinctada fucata were recorded only on control panels of steel and FRP materials. It is learned that the antifouling treatments showed good effect on controlling the oyster fouling on the studied boat building materials. (8) recorded only two species of oysters along Pondicherry Coast and they were $C$. madrasensis and Saccostrea cucullata. (11) recorded three species of oysters in Mumbai Coast. But (1) recorded only one species C.madrasensis in Pondichery Coast. 


\section{Ascidians species recorded on various test} panels

Ascidians are also important group of foulers in tropical waters. As far as wood is concerned, both control and copper nanocoated were recorded with ascidians while the antifouling painted panels showed good impact on controlling the attachement of ascidians. But in the case wooden panels of both treated and control of did not show any foulers from ascidians group. The FRP panels showed similar pattern of ascidians fouling as that of wood. (11) reported 10 species of ascidians species on the biofouling study panels in Mumbai Harbour.

In the present study only two species were recorded, this may be attributed to the tidal influence and food availability in the port area.

Barnacles were showed progressive increment by numbers during the soaking periods, irrespective of materials selected for study.

Two species of barnacles viz. B.amphitrite and B.variegates were recorded during the study period. Of these two species, B.amphitrite was dominant over other species.

Relatively high degree of barnacle fouling was observed in FRP panels.

The antifouling effect of copper nanocoated panels were found to be good when comparing to the effect of antifouling paint.

Poor biodiversity of barnacle species in the Thoothukudi port area was attributed to less food availability in the study area.

Both antifouling treatments under the present study, showed good effect in controlling the mussels. Two species of mussels, namely P.indica and Anomia spp were recorded in the control panels.
Two oyster species namely C.madrasensis and P.fucata were recorded in the control panels of studied boat building materials. Both, antifouling paint and nanocoating were showed good effect on controlling the oysters.

The commercial grade antifouling paint was found to be effective in controlling ascidian fouling in Thoothukudi waters. Copper nanocoating was much effective in controlling ascidians attachment selected boat building materials except in steel.

Copper nanocoating was found to be better in controlling the foulers in general compared to the commercial grade antifouling paints.

\section{Acknowledgement}

The authors are thankful to the Dean, Fisheries College and Research Institute, Tamil Nadu Fisheries University, Thoothukudi 628008, Tamil Nadu, India for rendering the facilities to conduct the present study. I wish to express my sincere thanks to Dr. G. Shakthinathan, Dean, Anna University, Tirunelveli campus, Tirunelveli, for providing facilities of nanocoating in this study. I would like to express my heartfelt gratitude to Dr. G. Subramanian, Scientist, and Mrs. P. Kalaiselvi Central Institute of Electrochemical Research Institute, for providing facilities of hanging of panels and their guidance in the period of study.

\section{References}

1. Anand Babu R, Yogamoorthi A, Siva Sankar R, Kaviarasan T, Distribution of Macro Fouling Community in the Coastal Region of Pondicherry, India. International Journal of Fisheries and Aquatic Sciences, 1(2): 147-149 (2012).

2. Jones G, The battle against marine biofouling: a historical review. In Advances in Marine Antifouling 
coatings and Technologies; Helio C., Yebra D.M.Y. (Eds) Woodshead Publishing: Cambridge, UK: 19-45 (2009).

3. Kelkar PG, Studies on diatoms from the offshore region of the Arabian Sea with special reference to fouling diatoms, Ph.D thesis, Goa University, India (1989).

4. Matias JR, Rabenhorst J, Mary A, Lorilla AA, Marine biofouling testing of experimental marine paints. Technical considerations on methods, site selection and dynamic tests. Proceedings of the SSPC 2003 Industrial protective coatings conference and exhibit in New Orleans, Louisiana (2003).

5. Mitbavkar S, Anil AC, Diatom Colonization on stainless steel panels in estuarine Waters of Goa, west coast of India. Indian J.Mar.Sci, 29: 273-6 (2000).

6. Pangu PN, Some ecological studies on the fouling communities in estuarine environments along the central west coast of India. Ph.D. Thesis, Goa University, Goa (1993).

7. Ramadan SHE, Kheirallah AM, AbdelSalam KHM, Marine fouling community in the Eastern harbour of Alexandria, Egypt compared with four decades of previous studies
Mediterranean Marine Science, 7 (2): 19 - 29 (2006).

8. Rajagopal S, Azariah J, Nair KVK, Ecology of fouling organisms in Edaiyur backwaters, Kalpakkam, Mahasagar, 23: 29-35(1990).

9. Satpathy KK, Nair KVK, Impact of power plant discharge on the physicochemical characteristics of Kalpakkam coastal waters, Mahasagar - Bulletin of National Institute of Oceanography, 23, 117-125(1990).

10. Sophia Rani S, Bragadeeswaran S, Prabhu K, Priyadharshini S, Infestation of Barnacle (Balanus amphitrite) in the mangrove environment of Vellar Estuary, Tamilnadu. World Journal of Fish and Marine sciences, 2(4): 307310 (2010).

11. Swami.BS Udhayakumar M, Seasonal influence on settlement distribution and diversity of fouling organisms at Mumbai harbour. Indian J. Marine Sciences, 39 (1): 57-67 (2010).

12. Tara Kandha B, Jeffrey J, Morell Satyanarayanan Rao K,Impacts of wood preservatives (CCA, CDDC, ACZA, ACQ and ACC) on the settlement and Growth of fouling organism. Proceedings of the onference on Environmental Impact of Presevative Treated wood. orlando FL, 8-11 (2004).

\section{How to cite this article:}

Archana, S., B. Sundaramoorthy, N. Neethiselvan and Jeyashakila, R. 2019. Impact of Nanocoating on Micro and Macro Foulers in FRP, Steel and Wooden Materials. Int.J.Curr.Microbiol.App.Sci. 8(08): 2908-2922. doi: https://doi.org/10.20546/ijcmas.2019.807.361 